\title{
APROXIMACIÓN AL PERFIL DEL ORIENTADOR EN LA ESO DE ARAGÓN
}

\author{
APPROACHING TO THE SCHOOL COUNSELOR'S PROFILE IN THE \\ SECONDARY OBLIGATORY EDUCATION IN ARAGÓN
}

\author{
Narciso García Nieto* \\ Universidad Complutense \\ Jacobo Cano Escoriaza** \\ Universidad de Zaragoza
}

\begin{abstract}
RESUMEN
El objetivo del artículo es analizar el perfil del orientador que trabaja en la Comunidad Autónoma de Aragón en el ámbito educativo en la ESO. Se recogen datos de más del 80\% de estos profesionales a través de un Cuestionario realizado específicamente para esta investigación sobre el Análisis sobre las Funciones de los Orientadores (CAFO) y se hace una aproximación a sus características más relevantes. Se incluyen variables personales - edad, sexo, provincia, formación inicial, de grado y postgrado-, variables profesionales - años de experiencia como orientadores, como docentes, situación laboral, dedicación y horario - y variables relacionadas con su Centro - titularidad, programas que imparte, miembros del Departamento de Orientación y alumnado que atiende-
\end{abstract}

Palabras clave: Perfil del orientador. Formación inicial. Funciones del orientador. Departamento de Orientación.

\section{ABSTRACT}

This paper aims at analyzing the professional profile of the school counsellors working in Aragon Secondary Schools. We collected data from over $80 \%$ of Aragon School counsellors by means of a questionnaire specially constructed for this research to analyze their tasks and to know their main characteristics. We include personal variables such as age, gender, region, or training. We also analyze professional skills, such as experience as counsellors and teachers, full/part time basis, or timeta-

\footnotetext{
* Catedrático de Orientación Educativa de la Universidad Complutense. Las principales líneas de investigación son: Atención a la diversidad, Orientación y Tutoría e Intervención Psicopedagogica. nargar@edu.ucm.es

** Profesor de la Universidad de Zaragoza desde 2002. Premio Extraordinario de Doctorado en Psicopedagogía de la Universidad Complutense. Tutor del Master de Terapia Familiar de la Universidad de Zaragoza. Orientador y terapeuta familiar acreditado por la FEAP. Las principales líneas de investigación están relacionadas con la Orientación, la Intervención Familiar, la Atención a la Diversidad y la Organización Escolar. jcano@unizar.es
} 
ble. Finally, we analyze variables related to the work place: public/private status, variety of programmes, number of people in the counselling department, and type of students.

Key words: School counsellors profile, initial training, counsellor roles, guidance department.

\section{Introducción}

Es de señalar que la investigación sobre la Orientación Educativa, especialmente a partir de la década de los noventa en la que con la LOGSE se le da un gran impulso, es creciente en nuestro país (Alonso, 1995; Álvarez y Bisquerra, 1996; Vélaz, 1998; Benavent, 1999 y 2003; Repetto, 2002; Soler, 2004). Sin embargo, se echan en falta estudios, pormenorizados, acerca de las características personales, profesionales y relativas a los Orientadores en cada una de las Comunidades Autónomas (Royo, 2002; Gomáriz et al., 2003; Menéndez, 2004) y, en este caso, carecemos de estudios relevantes en Aragón.

Señalamos alguna aportación relevante en el ámbito de la Orientación específicamente desarrolladas en Aragón. En primer lugar, la Orden de 22 de agosto de 2002 del Departamento de Educación y Ciencia del Gobierno de Aragón, relativa a la organización y el funcionamiento de los Centros Docentes Públicos de Educación Secundaria de la Comunidad Autónoma, se especifican las siguientes pautas de organización en el horario del orientador: Entre seis y nueve periodos lectivos dedicados a impartir materias optativas relacionadas con su especialidad y a la atención de grupos de alumnos que sigan programas específicos, según determine la Jefatura de Estudios; Horas de despacho para la atención de alumnado, familias, profesorado y preparación de materiales; Horas dedicadas a la coordinación y reuniones con equipo directivo y tutores; Una hora para reuniones de Departamento; Horas dedicadas a la coordinación con colegios públicos adscritos.

A continuación, exponemos la metodología seguidamente utilizada para recabar datos referentes al perfil del orientador en Aragón y las connotaciones específicas de éste en la Comunidad Autónoma.

\section{Método}

Vamos a describir algunos rasgos destacables de la muestra de los orientadores de la Comunidad Autónoma de Aragón que han respondido al Cuestionario de Análisis de las Funciones de los Orientadores (CAFO) que se les ha enviado. Dicho Cuestionario para la recogida de datos se refiere a la percepción que tienen sobre sus funciones en la ESO, constatando, a la vez, los resultados referidos a los datos que define un perfil general del orientador. El instrumento diseñado pretende clarificar el problema acotado en este artículo (Martínez, 2002; Rojas et al., 1998).

Algunas de las ventajas de la utilización de la encuesta son las siguientes: alta representatividad ya que nos permite llegar a todos los profesionales y calidad de la información disminuyendo la deseabilidad social al no influir el entrevistador. Hemos adaptado un proceso minucioso de seguimiento de las respuestas en las encuestas enviadas por correo (Robson, 1993).

Es de observar que el instrumento de referencia obtuvo un resultado muy positivo, mediante el Alfa de Cronbach $(0,97)$ con un índice de fiabilidad muy adecuado. Respecto a la 
validez de contenido, el instrumento, antes de llegar a la versión definitiva, lo han analizado un grupo de once expertos de reconocido prestigio, especialmente en los ámbitos de la docencia, la orientación y la investigación que han arrojado datos superiores al cuatro sobre cinco relativos a la claridad y pertinencia del Cuestionario propuesto.

Existen otros instrumentos que, de igual forma, han contribuido al desarrollo del conocimiento del desempeño profesional de los orientadores (Repetto et al., 1998; Valdivia et al., 1998; Royo, 2002).

Queremos señalar que el total de la población son 173 orientadores, si bien, 6 de ellos están trabajando en dos Centros. La respuesta de los 138 orientadores respecto a los 167 que hay en Aragón en la etapa de la ESO, representan un $81,4 \%$, dato que valoramos muy positivamente por su capacidad representativa y que refleja la buena disposición para colaborar en investigaciones que hacen referencia específicamente a su trabajo profesional y el seguimiento riguroso para la consecución de estos datos.

\section{Resultados}

En primer lugar, nos centramos en comentar y analizar diversas características de los participantes de la investigación. Analizaremos tres grupos de variables referidas, en primer lugar, a datos personales, continuando con los referidos al ejercicio profesional y, finalmente, al Centro.

\section{a) Datos personales}

Respecto a la edad, algo más de la mitad de los orientadores tienen entre 36-50 años, representando el $52,3 \%$, en la que se supone un buen grado de madurez y experiencia, al tiempo que se encuentran en un momento de estabilidad profesional, restando todavía más de una década como mínimo en su ejercicio profesional, para poder innovar y mejorar su práctica. La segunda franja de edad más numerosa corresponde a aquellos que se sitúan hasta los 35 años, abarcando el 30\%, dato que nos abre elevadas expectativas para el impulso de proyectos y programas renovadores en la escuela aragonesa. Y, en tercer lugar, tan sólo un 17,43\% que tienen más de 50 años, los cuales pueden aportar el bagaje de la experiencia sin ocultar los desánimos o ciertas dosis de realismo que reflejan las resistencias a cierto cambio o la crítica ante la Administración que en ocasiones ha podido dotar insuficientemente a estos profesionales de los recursos necesarios.

La mayoría de los orientadores son mujeres, representando un $65,44 \%$ frente al $34,56 \%$ de hombres. Se valora este dato, en la medida en que estos profesionales encarnan su quehacer profesional con la comunidad educativa relacionada con el periodo evolutivo de la adolescencia y, es preciso, un trabajo con enorme dosis de sensibilidad, de empatía y de autoridad.

La presente investigación se circunscribe a la Comunidad Autónoma de Aragón la cual tiene un reparto desigual en su territorio. Este aspecto también lo podemos constatar en el número de orientadores que hay en cada una de las provincias. Zaragoza es la provincia que centraliza la mayor parte de los servicios y, en este sentido, se concentran alrededor del $65 \%$ de los orientadores (89). En la provincia de Huesca hay un 18,38\% (25) y en Teruel un 
16,18\% (22). Estos datos nos hacen plantear la necesidad de impulsar la vertebración del territorio y la igualdad de oportunidades entre todos los escolares tanto del ámbito rural como del urbano, siendo preciso dotar a la escuela rural de los recursos y profesionales pertinentes para trabajar por una educación de calidad.

La formación recibida de los orientadores en ejercicio es muy heterogénea. La hemos dividido según los tipos de estudios oficiales que existen en la Universidad en la actualidad, según su duración y nivel. Hemos incluido, en primer lugar, la formación en la diplomatura de Magisterio - si tienen estos estudios o no, especificando la especialidad- y, en segundo lugar, hemos solicitado que señalasen la formación de licenciatura.

Hemos realizado una recodificación que queda de la siguiente manera:

a) Formación a nivel de maestro. Si son o no maestros.

b) Formación a nivel de licenciados. Hemos especificado entre las licenciaturas de Psicología y Pedagogía. Los psicopedagogos, de forma artificial y atendiendo al currículum de dichos estudios, los hemos incluido en la categoría de pedagogos.

Los orientadores que han realizado la diplomatura de Magisterio en cualquiera de sus diferentes especialidades representan el $35,29 \%$, frente al $64,71 \%$ que no son maestros. Esa tercera parte de profesionales con formación previa en esta diplomatura, puede ofrecer una visión muy realista y cercana de las situaciones que han estudiado o vivenciado en las primeras edades, sobre todo, teniendo en cuenta la importancia que la prevención tiene en la intervención psicopedagógica.

Con relación a la formación, hemos agrupado algunas categorías. No hemos diferenciado los diferentes planes de estudio de las licenciaturas, ni la forma como se denominan sino que hemos analizado dicha formación de los orientadores según hayan realizado Pedagogía, Psicología, Psicopedagogía o las dos primeras carreras universitarias.

En este sentido, podemos señalar que el porcentaje mayor corresponde a los orientadores que han realizado Psicología, siendo algo más de la mitad de ellos, un 53\%. Posteriormente, los de Pedagogía representan un $33,58 \%$. A más distancia, lógicamente por la novedad de la carrera y su trayectoria más breve, se encuentran aquellos que han realizado Psicopedagogía, que ni siquiera llegan al 10\% (8,96\%). Finalmente, el grupo que tiene tanto la licenciatura de Psicología como de Pedagogía representan un 4,48\%.

La mayoría de los orientadores no son doctores, representando un $79,1 \%$, frente al $20,9 \%$ que son doctores o están en proceso de serlo, ya que están realizando los cursos de doctorado o la tesis doctoral. Este porcentaje nada desdeñable puede ser una de las vías por las cuales la escuela aragonesa se favorezca de la necesaria investigación en materia de innovación educativa dada su previa experiencia, al tiempo que se requerirán medidas de la Administración Educativa para invertir los recursos precisos para hacer la necesaria reflexión acerca de la práctica educativa y la intervención que desde los profesionales de la orientación implementan en el día a día.

Esta variable referida a la formación en grado de máster o postgrado la hemos definido como aquellos estudios o cursos posteriores a la diplomatura o licenciatura y que tienen como objetivo complementar la formación académica y científica del profesional.

En este sentido, la hemos dividido en tres apartados: En primer lugar, formación relacionada con alguna de las funciones específicas del orientador; en segundo, la formación no 
relacionada con la profesión del orientador y en último lugar, los que carecen de formación de postgrado.

En la recodificación, hemos unido aquellos que no tienen formación con los que tienen algún tipo de máster pero no específicamente de temas vinculados a nuestro campo de trabajo.

Observando los datos, podemos señalar cómo el $28 \%$ de los orientadores ha realizado algún máster o postgrado relacionado directamente con su trabajo profesional. Tan sólo un $4,41 \%$ ha realizado algún otro máster pero no relacionado con sus funciones actuales como orientador, y el resto, un $66,18 \%$ no ha prolongado su formación con ningún máster. Nos parecen datos positivos de partida, dada la trayectoria y experiencia vital de muchos de los orientadores tal y como hemos señalado anteriormente, pero es preciso seguir aumentando la formación para dignificar y fundamentar lo más posible la intervención psicopedagógica.

A modo de síntesis, aportamos los datos en la siguiente tabla.

TABLA N ${ }^{\circ}$ 1: Variables de identificación: datos personales.

\begin{tabular}{|c|c|c|c|c|c|}
\hline \multirow{2}{*}{ 1. Edad } & \multicolumn{2}{|c|}{ Hasta 35} & & $36-50$ & Más de 50 \\
\hline & \multicolumn{2}{|l|}{$30,3 \%$} & & $52,3 \%$ & $17,4 \%$ \\
\hline \multirow{2}{*}{ 2. Sexo } & \multicolumn{3}{|c|}{ Hombre } & \multicolumn{2}{|r|}{ Mujer } \\
\hline & \multicolumn{3}{|c|}{$34,6 \%$} & \multicolumn{2}{|r|}{$65,4 \%$} \\
\hline \multirow{2}{*}{ 3. Provincia } & \multicolumn{2}{|c|}{ Huesca } & \multicolumn{2}{|r|}{ Teruel } & Zaragoza \\
\hline & \multicolumn{2}{|l|}{$18,4 \%$} & & $16,2 \%$ & $65,4 \%$ \\
\hline \multirow{2}{*}{$\begin{array}{l}\text { 4. Formación } \\
\text { básica }\end{array}$} & Pedagogía & \multicolumn{2}{|c|}{ Psicología } & Psicopedagogía & Psicología y Pedagogía \\
\hline & $33,6 \%$ & \multicolumn{2}{|c|}{$53 \%$} & $9 \%$ & $4,4 \%$ \\
\hline \multirow{2}{*}{ 5. Doctorado } & \multicolumn{3}{|c|}{ Doctor o en proceso } & \multicolumn{2}{|c|}{ No doctor } \\
\hline & \multicolumn{3}{|c|}{$20,9 \%$} & \multicolumn{2}{|r|}{$79,1 \%$} \\
\hline \multirow{2}{*}{ 6. Master } & \multicolumn{2}{|c|}{ Si relacionados } & \multicolumn{2}{|c|}{ No relacionados } & No tiene \\
\hline & \multicolumn{2}{|l|}{$28,5 \%$} & & $4,7 \%$ & $66,8 \%$ \\
\hline
\end{tabular}

\section{b) Datos sobre el ejercicio profesional}

En este segundo apartado, nos referimos a los datos que nos ofrecen los orientadores específicamente sobre su ejercicio profesional. En este sentido, comenzamos comentando sobre los años de experiencia en el Departamento de Orientación del Centro en el que se encuentra el orientador en el momento de rellenar el Cuestionario. Aquellos que han señalado que tienen menos de medio año, hemos considerado que no tenían ningún año de experiencia; aquellos que han señalado más de seis meses de experiencia, hemos considerado que tenían un año de experiencia. 
La recodificación la hemos hecho según la siguiente agrupación: a) Ningún año de experiencia, b) de 1-5 años, c) más de 5 años.

La mitad de los orientadores tiene entre 0-5 años de experiencia en el Departamento de Orientación en el que actualmente están, dato que refleja la relativa juventud del reconocimiento de los orientadores en la práctica educativa aragonesa. Ligeramente por debajo, un 44,78\% tiene entre 6-15 años de experiencia. Finalmente, los más veteranos, es decir, aquellos orientadores que están trabajando en el mismo Departamento desde hace más de 15 años representan un 5,22\%.

Aproximadamente la mitad de los orientadores $(48,78 \%)$ no ha trabajado ningún año en un Departamento de Orientación de otro Centro. Un 31,71\% ha trabajado entre 1-5 años en otros Departamentos, y casi un 20\% lo ha hecho entre 6-10 años. En este sentido, podemos señalar aquellos orientadores que trabajan en la red pública y que han ido realizando interinidades en diversos IES, pero resulta llamativo que algo más de la mitad de los orientadores ha estado en diversos Centros, teniendo, cuando menos, una doble lectura, positiva por un lado, en cuanto al enriquecimiento que supone trabajar en ámbitos de trabajo diversos, pero, negativa en la medida en que no contribuye de la manera más eficaz posible a la consolidación de la práctica de la orientación en un Centro determinado.

Respecto a la experiencia docente actual de los orientadores, relacionado con las materias propias (Psicología, Transición a la Vida Adulta y Activa, Programas Específicos de Estrategias de Aprendizaje, etc.), un 40\% no imparte este tipo de asignaturas, frente a un $37 \%$ que imparte una de ellas y un $22 \%$ que da clases de más de dos asignaturas.

Las asignaturas relacionadas a las que nos referimos con esta pregunta corresponden fundamentalmente a Ética o Filosofía. En este sentido, un $80 \%$ aproximadamente no imparte ninguna de estas materias, frente al $18,66 \%$ que están dando clase de una de ellas y tan sólo dos orientadores están dando más de dos asignaturas.

Las asignaturas no relacionadas hacen referencia a cualquiera que no hayan entrado en las dos categorías anteriores de asignaturas propias - Psicología, Transición a la Vida Adulta y Activa, Programas Específicos de Estrategias de Aprendizaje, etc.- y relacionadas - Ética o Filosofía- Hemos visto que hay orientadores que imparten, por ejemplo, Geografía, Lengua Castellana y Literatura, Cultura Clásica o Matemáticas.

Tenemos que señalar, tal y como avanzábamos anteriormente con relación a las asignaturas de Ética o Filosofía, que también cerca del $80 \%$ no imparte ninguna asignatura no relacionada. Al menos una de las asignaturas variadas que citábamos en el párrafo anterior, la impartían un $14,18 \%$ y un $7,46 \%$ más de dos. Hemos recogido la diversidad de opiniones de los orientadores a este respecto. Mientras unos señalan el valor positivo de complementar su labor orientadora con la docencia para conocer, de una forma eminentemente directa, la realidad educativa y tener un status o experiencia docente más igualitario con el resto del profesorado para que éste lo perciba más colaborativo; otros remarcan lo negativo que resulta además de la gran carga de trabajo y diversidad de funciones, añadir la parte docente, que resta tiempo a otras funciones especializadas relevantes como el diagnóstico y la evaluación psicopedagógica. En Aragón, la Administración Educativa marca una horquilla entre las seis y nueve horas de dedicación a la docencia.

En relación con el número de asignaturas impartidas en el pasado, un 57,36\% no ha impartido anteriormente ni Psicología, ni Transición a la Vida Adulta y Activa ni un programa específico de Estrategias de Aprendizaje. Casi un 25\% ha impartido una de ellas y un 
$17,83 \%$ lo ha hecho anteriormente con más de una. Un $83 \%$ de los orientadores no ha impartido anteriormente ninguna asignatura relacionada, como Ética o Filosofía, frente al $16,28 \%$ que ha impartido una de ellas. Tan sólo un orientador, ha impartido anteriormente las dos asignaturas. Con relación a las asignaturas que no están incluidas en los anteriores dos apartados, observamos cómo un $65 \%$ no ha impartido ninguna anteriormente. Un $20 \%$ aproximadamente ha dado una asignatura no relacionada específicamente con su perfil de orientador y casi un $15 \%$ lo ha hecho con más de una asignatura.

Recogemos los años que el orientador ha ejercido algunos de los siguientes puestos:

a) Dentro del Centro: Director, Jefe de Estudios, Coordinador, Tutor, Profesor, Secretario y Jefe del Departamento de Orientación.

b) Otras funciones: Ejercicio como asesor en un Centro de Profesores y Recursos, en un Equipo de Orientación Educativa y Psicopedagógica y/o en un Equipo de Atención Temprana.

La recodificación la hemos hecho según la siguiente triple agrupación: En primer lugar, ningún año de experiencia, en segundo, entre 1-5 años, y, finalmente, más de 5 .

Casi un $85 \%$ de orientadores no ha ejercido de Director, frente al $11,1 \%$ que lo ha hecho entre 1-5 años y un 4,44\% que ha sido más de 5 años. Un $90 \%$ no ha ejercido de Jefe de Estudios, frente al 9\% aproximadamente que ha sido entre 1-5 años. Tan sólo dos orientadores, han ejercido como Jefe de Estudios más de 5 años. Algo más del $90 \%$ no ha sido Coordinador. El 6\% ha ejercido entre 1-5 años y 4 lo han hecho más de cinco años, representando el 3\%. Un 67\% de los orientadores no ha ejercido de tutor, mientras que el 17,29\% lo ha hecho entre 1-5 años y otro $15 \%$ ha ejercido más de 5 . Algo más del $50 \%$ no ha ejercido de profesor nunca (53,73\%), mientras que un 14,18\% lo ha hecho entre 1-5 años. Por su parte, el 32\% de orientadores ha ejercido de profesor más de 5 años. Casi la totalidad de orientadores no ha ejercido nunca de Secretario en el Centro (95,52\%). Tan sólo un 3\% lo ha hecho entre 1-5 años y dos orientadores que representan el 1,5\% han sido Secretario más de 5 años. La inmensa mayoría de orientadores, es decir, un 97,78\% no ha ejercido de asesor en un Centro de Profesores y Recursos. Tan sólo dos orientadores lo han hecho entre 1-5 años, y un único orientador, más de 5 años. Un $70 \%$ de orientadores no ha trabajado nunca en un Equipo de Orientación, mientras que casi un 25\% lo ha hecho entre 1-5 años. Por su parte, un $6 \%$ ha trabajado en dichos Equipos más de 5 años. Un 95,56\% no ha trabajado en el ámbito de la atención temprana nunca. Tan sólo tres orientadores han trabajado entre 1-5 años y otros tres lo han hecho más de 5 años. El grupo mayoritario de orientadores, algo más de la mitad, ha ejercido de Jefe del Departamento de Orientación entre 4-10 años, representando un $52,6 \%$. Por su parte, se sitúan en torno al $15 \%$ de orientadores - unos 20 aproximadamente-, los que han asumido las funciones de Jefe entre 1-3 años, entre 11-20 años o no lo ha hecho nunca. Por último, 5 orientadores $(3,70 \%)$ han sido Jefes del Departamento durante más de 20 años.

Remarcamos los porcentajes de los orientadores que han ejercido como profesores y/o tutores, ya que les ha podido dar una visión muy realista del panorama educativo y de las relaciones existentes de forma directa con el alumnado, con sus familias y con los compañeros del Claustro.

La estabilidad en el trabajo es una situación importante que puede modular las diferentes percepciones que pueden tener los orientadores acerca de sus funciones. Como aspecto muy positivo, remarcamos cómo la mayoría de los orientadores, un $83,7 \%$, manifiesta ser 
fijo o definitivo en su puesto de trabajo, frente a un 16,3\% que su situación es de temporalidad o provisionalidad.

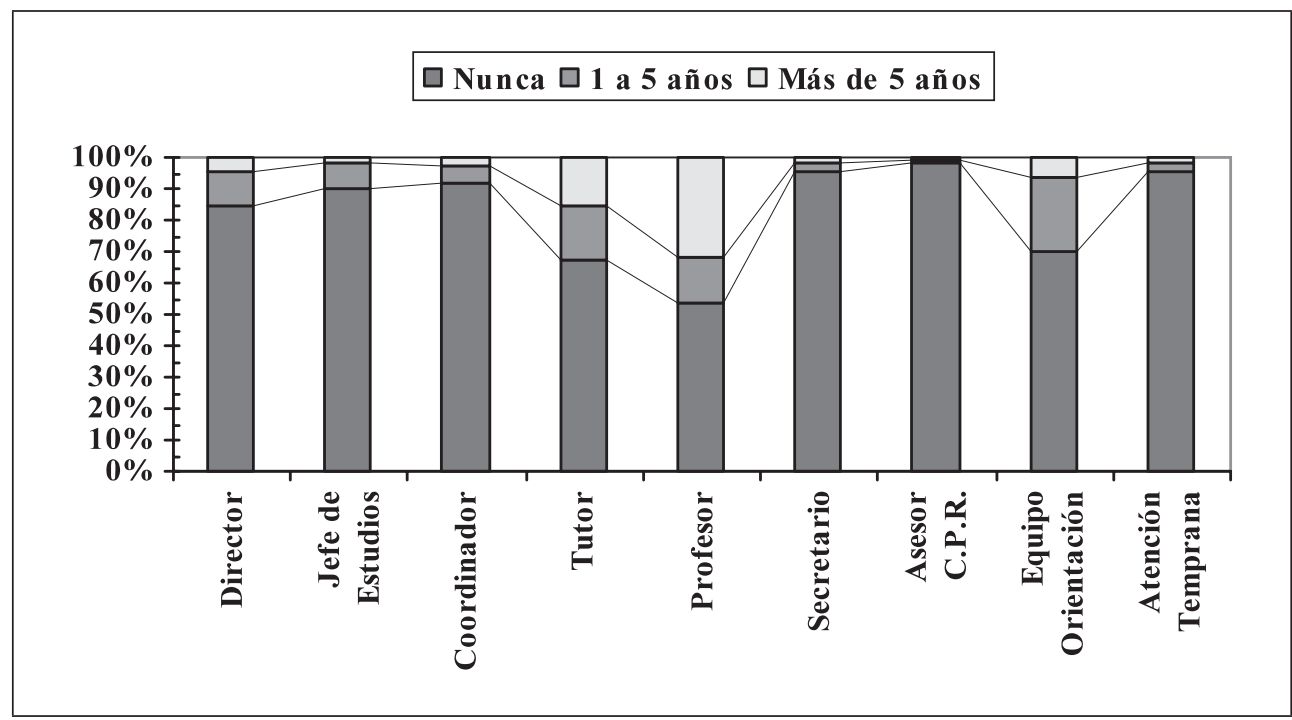

GRÁFICO 1.

Resumen de los años de ejercicio en distintas responsabilidades.

Por otro lado, la dedicación se ha definido como una variable que hace referencia al número de horas de la jornada, especificando si es a tiempo completo o parcial — conviene recordar que los orientadores de los Centros concertados desempeñan una hora de orientación por cada una de las unidades que tiene el Centro en la etapa de la Educación Secundaria Obligatoria- Tienen dedicación a tiempo completo el $67,4 \%$ de orientadores frente a un $32,6 \%$ que disponen de una jornada a tiempo parcial para desarrollar sus funciones.

Por su parte, en la variable horario hacemos referencia a las horas semanales dedicadas a las siguientes actividades: orientación/tutoría/docencia/otras.

Casi la mitad de los orientadores (48\%) dedica entre 6-12 horas a la orientación. Algo más de un $20 \%$, cerca de 30 Centros, dedican a la orientación tan sólo entre 2-5 horas. Si sumamos estos dos datos anteriores, podríamos decir que en torno al $70 \%$ de los orientadores dedica específicamente a la orientación menos de 12 horas. Por otro lado, un $20 \%$ dedica entre 13-20 horas. Por último, un 10\% dedica más de 20 horas. Un $60 \%$ de orientadores no tiene ninguna tutoría a su cargo, frente a un $14,5 \%$ que dedica una o dos horas. Por su parte, casi un $25 \%$ dedica más de dos horas a la tutoría. Podría interpretarse este dato como aquellos que dedican a la atención personal del alumnado o de las familias, y no sólo a la tutoría considerada de forma grupal. Algo más de un $26 \%$ de orientadores no dedica ninguna hora a impartir docencia (35). Un 13,4\% dedica entre 1-5 horas a impartir clase. El grupo mayoritario, un $40 \%$, corresponde a aquellos que tienen entre 6-9 horas de docencia. Finalmente, un $20 \%$ (27) de los orientadores imparten clase más de 9 horas. Exponemos la siguiente tabla con la finalidad de clarificar los datos más relevantes expuestos con anterioridad. 
TABLA No 2: Variables de identificación: datos sobre el ejercicio profesional.

\begin{tabular}{|c|c|c|c|c|c|c|}
\hline \multirow{2}{*}{\multicolumn{3}{|c|}{$\begin{array}{l}\text { 1. Años en el actual Departamento de } \\
\text { Orientación }\end{array}$}} & $0-5$ & \multicolumn{2}{|c|}{$6-15$} & $>15$ \\
\hline & & & $50 \%$ & \multicolumn{2}{|c|}{$44,8 \%$} & $5,2 \%$ \\
\hline \multirow{2}{*}{\multicolumn{3}{|c|}{$\begin{array}{l}\text { 2. Años en el Departamento de Orientación } \\
\text { de otros Centros }\end{array}$}} & $0-1$ & \multicolumn{2}{|c|}{$1-5$} & $6-10$ \\
\hline & & & $48,8 \%$ & \multicolumn{2}{|c|}{$31,7 \%$} & $19,5 \%$ \\
\hline \multicolumn{3}{|c|}{$\begin{array}{l}\text { 3. Asignaturas propias que imparte en la } \\
\text { actualidad }\end{array}$} & Ninguna & \multicolumn{2}{|c|}{ Una } & Más de una \\
\hline Propias & Relacionadas & No relacionadas & $40,3 \%$ & \multicolumn{2}{|c|}{$37,3 \%$} & $22,4 \%$ \\
\hline \multicolumn{3}{|c|}{$\begin{array}{l}\text { 4. Asignaturas propias que ha impartido } \\
\text { anteriormente }\end{array}$} & Ninguna & \multicolumn{2}{|c|}{ Una } & Más de una \\
\hline Propias & Relacionadas & No relacionadas & $57,4 \%$ & \multicolumn{2}{|c|}{$24,8 \%$} & $17,8 \%$ \\
\hline \multirow{4}{*}{\multicolumn{3}{|c|}{ 5. Años de ejercicio en distintos puestos }} & \multicolumn{2}{|c|}{$\begin{array}{l}\text { Director/Jefe } \\
\text { Estudios }\end{array}$} & \multicolumn{2}{|r|}{ Secretario } \\
\hline & & & \multicolumn{2}{|c|}{ Coordinador } & \multicolumn{2}{|c|}{ Asesor C.P.R. } \\
\hline & & & \multicolumn{2}{|c|}{ Tutor } & \multicolumn{2}{|c|}{ Equipo Orientación } \\
\hline & & & \multicolumn{2}{|c|}{ Profesor } & \multicolumn{2}{|c|}{ Atención Temprana } \\
\hline \multirow{2}{*}{\multicolumn{3}{|c|}{ 6. Situación laboral }} & \multicolumn{2}{|c|}{ Fijo o definitivo } & \multicolumn{2}{|c|}{ Temporal o provisional } \\
\hline & & & \multicolumn{2}{|c|}{$83,7 \%$} & & $16,3 \%$ \\
\hline \multirow{2}{*}{\multicolumn{3}{|c|}{ 7. Dedicación }} & \multicolumn{2}{|c|}{ Tiempo completo } & \multicolumn{2}{|c|}{ Tiempo parcial } \\
\hline & & & \multicolumn{2}{|c|}{$67,4 \%$} & \multicolumn{2}{|r|}{$32,6 \%$} \\
\hline
\end{tabular}

\section{c) Datos del Centro}

Los datos del Centro que incluimos en este epígrafe se refieren a su tipología según los fondos recibidos, la antigüedad del Departamento de Orientación, la composición del mismo, los niveles/ciclos que atiende, el alumnado, los habitantes del municipio y los grupos de que dispone.

De los que han contestado el Cuestionario, casi el 50\% de orientadores trabajan en Institutos de Educación Secundaria, frente a un $46 \%$ que lo hace en Centros Concertados. El porcentaje de orientadores que trabajan en Centros Privados es de un 4,44\%, es decir, 6 orientadores.

Veamos algunos datos interesantes acerca de la trayectoria en la implantación de los Departamentos de Orientación. Casi la mitad de éstos, un 48,1\% tiene entre 6-12 años de antigüedad en el Centro y un 15\% tiene más de doce años. Los que son de reciente creación, es decir, entre 1-5 años, suponen un 26,4\%. Finalmente, un 10\% de los Departamen- 
tos de Orientación no tienen ningún año de trayectoria en el Centro y han sido creados en el año actual. El máximo de años de permanencia del psicopedagogo en un mismo Centro está en torno a los diez, coincidiendo en este caso tres orientadores con la creación de dichos Departamentos.

La composición del Departamento de Orientación hace relación al número de miembros que están incluidos en él y se intenta concretar la presencia o no de los siguientes especialistas: Especialista en Psicología, Pedagogía y/o Psicopedagogía; Especialista en los ámbitos Tecnológico o Científico y Social o Lingüístico; Audición y Lenguaje; Pedagogía Terapéutica y del Cuerpo de Profesores Técnicos de Formación Profesional. Un 26,5\% de Departamentos de Orientación (35 Centros) está compuesto exclusivamente por el orientador —psicólogo, pedagogo o psicopedagogo-. Un 19\% tiene de 1,5-3 miembros. Alrededor del 37\% - 49 Centros - el Departamento tiene entre 4-6 miembros. Finalmente, un $17,42 \%$ - 23 Centros - tiene más de 6 miembros. El profesor de Formación y Orientación Laboral —FOL, destinado a los Ciclos Formativos y al Programa de Garantía Social- representa un 21,15\% de los centros. El Maestro de Pedagogía Terapéutica - P.T., o Profesor de Apoyo - aparece en un 53,85\% de los casos. Mientras que el Maestro de Audición y Lenguaje -A.L. o Logopeda-, forma parte del Departamento de Orientación en un $15,38 \%$ de los centros de la muestra. Es decir, tan sólo en ocho centros de los cuarenta y siete que afirman tener escolarizados alumnos con necesidades educativas especiales, existe Maestro especialista en Audición y Lenguaje. La figura del profesor de Educación Compensatoria forma parte del D.O. de los centros encuestados en un 19,23\% de los mismos. El Fisioterapeuta, como especialista en la atención al alumno con necesidades de tipo físico o motor, aparece en el 3,85\% de los centros participantes. Otros profesionales que aparecen aisladamente en algunos centros son: Asistente o trabajador social en tres centros; auxiliares técnicos educativos en dos centros; profesor técnico de servicios a la comunidad en dos centros; maestro de garantía social —área de formación básica - e intérprete de lengua de signos en un Centro. Los profesionales que aparecen en menor proporción se relacionan directamente con el ámbito de Atención a la Diversidad. Por un lado, la presencia de Maestros de Apoyo a la Integración de los alumnos con necesidades educativas especiales -Pedagogía Terapéutica, Audición y Lenguaje, Fisioterapeuta, Asistente Social, Intérprete de Lengua de Signos - no está generalizada en los Departamentos de Orientación de los centros de la muestra. Por otro lado, tampoco los Profesores que han de colaborar en los Programas de Diversificación y los Programas de Garantía Social —Profesores de Ámbito, Maestros de Formación Básica, Formación y Orientación Laboral- aparecen en muchos de los centros que desarrollan estos programas.

Con relación a los Niveles/Ciclos/Etapas que atiende el Departamento de Orientación, todos atienden a la Educación Secundaria Obligatoria. 55 atienden Bachillerato, 31 lo hacen en Ciclos Formativos de Grado Medio, 27 en Ciclos Formativos de Grado Superior y 35 en Programas de Garantía Social. Por otra parte, 8 Departamentos también se dedican a la Educación Infantil y 7 a la Educación Primaria. Prácticamente la mitad de los Centros cuyos orientadores han contestado el Cuestionario tiene menos de 300 alumnos. Otra tercera parte de ellos, los consideramos medianos ya que tiene entre 301-700 alumnos, mientras que el $16,4 \%$ tiene más de 700 .

Respecto a los habitantes del municipio, casi la mitad de los orientadores de la muestra se ubica en Zaragoza, representando un $46,67 \%$. A continuación, se sitúan uno de cada cinco orientadores cuyo Centro está en municipios entre 7.001-20.000 habitantes y un 17\% en 
los de 2.001-7.000. Finalmente, el resto de grupos, es decir, los de Huesca capital, los de Teruel capital y los de aquellos municipios de menos de 2.000 habitantes, han respondido siete orientadores en cada sector, lo que engloba un 5,2\%. La intervención de los orientadores es muy amplia de acuerdo también a la variedad de Programas implementados que existen en cada uno de los Centros, con el objetivo prioritario de establecer medidas para la necesaria atención a la diversidad. Para describir esta realidad, señalaremos que el $60 \%$ desarrolla algún Programa de Diversificación Curricular, de uno o de dos años; y el 43,5\% algún Programa de Educación Compensatoria. Casi una tercera parte de los orientadores señala que en su Centro se imparte algún Programa de Garantía Social y apenas uno de cada cinco orientadores, un 18,3\%, afirma que en su Centro no existe ningún grupo.

Incluimos la siguiente tabla que nos permite visualizar datos referidos a los Centros.

TABLA N ${ }^{\circ}$ 3: Variables de identificación: datos del centro.

\begin{tabular}{|c|c|c|c|c|c|c|c|c|}
\hline \multirow{4}{*}{$\begin{array}{l}\text { 1. Tipo de Centro } \\
\text { 2. Años de antigüedad del } \\
\text { Dpto. de Orientación en } \\
\text { el Centro }\end{array}$} & \multicolumn{3}{|c|}{ Público } & \multicolumn{2}{|c|}{ Concertado } & \multicolumn{3}{|c|}{ Privado } \\
\hline & \multicolumn{3}{|c|}{$49,6 \%$} & \multicolumn{2}{|c|}{$45,9 \%$} & \multicolumn{3}{|c|}{$4,4 \%$} \\
\hline & \multicolumn{2}{|c|}{ Ningún año } & \multicolumn{2}{|c|}{ 1-5 años } & \multicolumn{2}{|c|}{ 6-12 años } & \multicolumn{2}{|c|}{$>12$ años } \\
\hline & \multicolumn{2}{|c|}{$10,4 \%$} & \multicolumn{2}{|r|}{$26,4 \%$} & \multicolumn{2}{|l|}{$48,1 \%$} & \multicolumn{2}{|c|}{$15,1 \%$} \\
\hline \multirow{2}{*}{$\begin{array}{l}\text { 3. Miembros del Dpto. de } \\
\text { Orientación }\end{array}$} & \multicolumn{2}{|l|}{1} & \multicolumn{2}{|r|}{$1,5-3$} & \multicolumn{2}{|l|}{ 4-6 } & \multicolumn{2}{|c|}{$>6$} \\
\hline & \multicolumn{2}{|c|}{$26,5 \%$} & \multicolumn{2}{|r|}{$18,9 \%$} & \multicolumn{2}{|l|}{$37,1 \%$} & \multicolumn{2}{|c|}{$17,4 \%$} \\
\hline \multirow{2}{*}{$\begin{array}{l}\text { 4. Niveles/Ciclos que } \\
\text { atiende el Dpto. de } \\
\text { Orientación }\end{array}$} & \multicolumn{4}{|c|}{ Infantil y Primaria } & \multicolumn{4}{|c|}{ ESO y Bachillerato } \\
\hline & \multicolumn{4}{|c|}{ Ciclos Formativos de Grado Superior } & \multicolumn{4}{|c|}{ Programas de Garantía Social } \\
\hline \multirow{2}{*}{$\begin{array}{l}\text { 5. Alumnado que atiende } \\
\text { el Dpto. de Orientación }\end{array}$} & \multicolumn{3}{|c|}{ Pequeño $(<300)$} & \multicolumn{2}{|c|}{ Mediano $(301-700)$} & \multicolumn{3}{|c|}{ Grande $(>700)$} \\
\hline & \multicolumn{3}{|c|}{$49,3 \%$} & \multicolumn{2}{|c|}{$34,3 \%$} & \multicolumn{3}{|c|}{$16,4 \%$} \\
\hline \multirow{2}{*}{$\begin{array}{l}\text { 6. Habitantes del } \\
\text { municipio }\end{array}$} & $<2000$ & $20 C$ & $1-7000$ & $7001-20000$ & $20001-40000$ & 4000 & -60000 & $>60000$ \\
\hline & $5,2 \%$ & & $17 \%$ & $20,7 \%$ & $5,2 \%$ & & $2 \%$ & $46,7 \%$ \\
\hline & $\begin{array}{r}\text { P. D } \\
\text { Curric }\end{array}$ & & P. Ed & Compensat. & P. Garantía S & cial & Ningú & n grupo \\
\hline 7. Atención a diversos & 60,3 & & & $43,5 \%$ & $31,3 \%$ & & & ,3\% \\
\hline & Integra & ión & & UIIE & Desdobles/Ap & byos & & rios \\
\hline & 10,7 & & & $10,7 \%$ & $6,1 \%$ & & & $6 \%$ \\
\hline
\end{tabular}

\section{Conclusiones}

Somos conscientes de las limitaciones de este estudio, al circunscribirse únicamente a una de las Comunidades Autónomas. Sin embargo, hacemos constar que una de las fortalezas de este trabajo, es la alta tasa de respuesta de los orientadores de Aragón que se sitúa en más del $80 \%$. Si bien, sería deseable generalizar el trabajo a otros lugares, como futura línea de investigación, que incorporase de forma pormenorizada el análisis del perfil del orientador en el ámbito educativo, junto a la implementación de las diversas funciones encomendadas. De igual modo, es preciso seguir profundizando en la investigación referida no sólo a los orientadores del ámbito educativo y ampliando la circunscripción más allá de 
la ESO, incorporando el papel de la TIC, la participación de toda la comunidad educativa así como la evaluación de la calidad de la práctica orientadora.

\section{Perfil destacable del Orientador de la Comunidad Autónoma de Aragón}

Los datos más relevantes del perfil profesional de esta muestra, manifiestan que el orientador mayoritariamente es mujer (64\%), entre 36 y 50 años (42 años de media), trabajando en un IES (50\%), teniendo la mitad de ellas cuenta con una experiencia de entre 0-5 años en el Departamento de Orientación, en el que actualmente están y otra mitad ha estado en diversos Centros ejerciendo y con una formación de postgrado una cuarta parte de ellas. Más del 80\% tiene un puesto definitivo o fijo, aunque están a tiempo completo el $67,4 \%$ y el $70 \%$ dedica menos de 12 horas a la semana a la orientación — aspectos que habría que clarificar según sean IES o Centros Concertados-, ejerciendo a su vez la Jefatura del Departamento salvo un $15 \%$ que no lo hace. Prácticamente la mitad de los orientadores tiene más de 300 alumnos a su cargo y el 16\% de éstos más de 700 alumnos. Casi una tercera parte ha trabajado anteriormente en un Equipo de Orientación o ha ejercido de tutor/a y casi la mitad de profesor.

\section{El Departamento de Orientación en la Comunidad Autónoma de Aragón}

En relación a los Departamentos de Orientación —un 26,5\%, 35 Centros- está compuesto exclusivamente por el orientador — psicólogo, pedagogo o psicopedagogo- y el 60\% desarrolla algún Programa de Diversificación Curricular, el 43,5\% algún Programa de Educación Compensatoria, casi una tercera parte de los orientadores señala que en su Centro se imparte algún Programa de Garantía Social y apenas uno de cada cinco orientadores, un $18,3 \%$, afirma que en su Centro no existe ningún grupo específico de los señalados anteriormente.

\section{Implicaciones prácticas}

Por último, es importante señalar algunas implicaciones prácticas que se derivan, que van en la línea de incrementar la formación de postgrado y actualización de los orientadores para responder a las demandas actuales; reducir el porcentaje de Centros que no dispone de grupos específicos para atender a la diversidad del alumnado y su personal correspondiente en ciertos casos, así como disminuir la ratio orientador-alumnado; e incrementar la dedicación específica de horas de orientación a la semana por parte de estos profesionales.

\section{Referencias bibliográficas}

Alonso Tapia, J. (1995). Orientación educativa. Teoría, evaluación e intervención. Madrid: Síntesis. Álvarez, M. y Bisquerra, R. (1996). Manual de orientación y tutoría. Barcelona. Praxis.

Benavent, J. A. (1999). "La orientación psicopedagógica en el umbral del siglo XXI: Una mirada al futuro". Revista Española de Orientación y Psicopedagogía, 10 (17), 53-62. 
Benavent, J. A. (2003). "Reflexiones sobre el futuro de la orientación psicopedagógica inmersa en una encrucijada sociocultural”. Revista Española de Orientación y Psicopedagogía, 14 (1), 41-59.

Gomáriz, Ma A. et al. (2003). "Los Departamentos de Orientación en la región de Murcia: orientación y atención a la diversidad". Revista Española de Orientación y Psicopedagogía, 14 (2), 129-147

Martínez Olmo, F. (2002). El cuestionario. Un instrumento para la investigación de las ciencias sociales. Barcelona: Laertes Psicopedagogía.

Menéndez, E. (2004). "Evolución de los servicios de orientación en la Comunidad Autónoma de Galicia. Situación actual”. Revista Española de Orientación y Psicopedagogía, 15 (2), 401-411.

Repetto, E. et al. (1998). "Validación del Cuestionario español de formación del personal que trabaja en orientación basada en competencias". Revista Española de Orientación y Psicopedagogía, 9 (15), 57-85.

Repetto, E. (2002). Modelos de orientación e intervención psicopedagógica. Madrid: UNED.

Robson, C. (1993). Real world research. A resource for social scientist and practioner researchers. Oxford: Blackwell.

Rojas Tejada, A. J. et al. (1998). Investigar mediante encuestas. Fundamentos teóricos y aspectos prácticos. Madrid: Síntesis.

Royo, F. (2002). "Desempeño profesional de los orientadores en los Institutos de Educación Secundaria de Salamanca. Creación de un instrumento de valoración y autovaloración”. Revista Española de Orientación y Psicopedagogía, 13 (1), 31-50.

Soler, J. L. (Coord.) (2004). Orientación y tutoría. Actas del Congreso Internacional Asociación Aragonesa de Psicopedagogía. Zaragoza: Mira Editores.

Valdivia, C. et al. (1998). La orientación y tutoría en centros educativos: Cuestionario de evaluación $y$ análisis tutorial (CEAT. $2^{\mathrm{a}}$ ed.). Bilbao: Mensajero.

Vélaz de Medrano, C. (1998). Orientación e intervención psicopedagógica. Concepto, modelos, programas y evaluación. Málaga: Aljibe.

Fecha de recepción: 20-09-07

Fecha de revisión: 10-06-08

Fecha de aceptación: 10-09-08 